\title{
EL ECOTURISMO: ¿Una aspirina para el campo?
}

\section{ECOTURISMO: UMA ASPIRINA PARA O CAMPO?}

\section{ECOTOURISM: Aspirin for the countryside?}

\author{
Catherine Héau Lambert \\ Instituto Nacional de Antropología e História - ENAH/INAH. \\ Circuito Mario de la Cueva. Ciudad Universitaria, CP 04510 \\ México, D.F. México. \\ E-mail: catherineheau@gmail.com
}

\section{Resumen}

En lugar de subsidiar económicamente a las comunidades rurales pobres de las Bahías de Huatulco, Oaxaca, en nombre de la justicia social, el Estado mexicano las responsabiliza por su supervivencia convirtiéndolas al turismo rural o turismo comunitario, sistema económico donde la comunidad se emplea a sí misma en lugar de salir a vender su fuerza de trabajo. Es la alternativa neo-liberal para seguir siendo campesino. En el ámbito del turismo, la ecología se ha desvinculado de la idea utópica de contra-cultura anti consumista y anti materialista, para dedicarse ahora a la mercantilización y folklorización de la naturaleza y la cultura. Las comunidades rurales se han vuelto empresas de auto-empleo que proveen a las grandes agencias hoteleras con excursiones exóticas y fungen como empresas de outsourcingque trabajan para las agencias de viajes, dueñas de los medios de transporte. A respecto del ecoturismo como practicado en Huatulco, él ha dejado de ser un concepto alternativo para volverse una actividad complementaria de la industria del turismo.

Palabras clave: Ecoturismo y neoliberalismo. Exotismo e industria del turismo. Comunidades rurales y folklorización. Bahías de Huatulco-México.

\section{Resumo}

Em vez de subsidiar economicamente as comunidades rurais pobres de Baías de Huatulco, Oaxaca, em nome da justiça social, o Estado mexicano faz avanços em termos de desenvolvimento ao transferir a responsabilidade de sobrevivência às próprias comunidades, convertendo-as ao turismo rural ou turismo comunitário; um sistema econômico onde a comunidade se autoemprega em vez de vender sua força de trabalho. É a alternativa neoliberal para continuar sendo camponês. No âmbito do turismo, a ecologia tem se desvinculado da ideia utópica de contracultura anticonsumista e antimaterialista para se dedicar agora à mercantilização e "folclorização" da natureza e da cultura. As comunidades rurais se tornaram empresas de autoemprego terceirizadas contratadas para prover excursões exóticas às grandes agências hoteleiras e atuar como consultoras subcontratadas para trabalhar para as agências de viagens proprietários dos meios de transporte. Levando-se em consideração o ecoturismo como praticado em Huatulco, este tem deixado de ser um conceito alternativo para se transformar em uma atividade complementar da indústria do turismo.

Palavras-chave: Ecoturismo e neoliberalismo. Exotismo e indústria do turismo. Comunidades rurais e folclorização. Baías de Huatulco-México. 


\section{Abstract}

Instead of financially subsidizing poor rural communities in Huatulco Bays, Oaxaca, in the name of social justice, the Mexican state has made them responsible for their own survival by converting them to rural o community-based tourism, an economic system in which the community is self-employed instead of selling their labour force. This is the neo-liberal alternative to continue being a peasant. In the field of tourism, ecology has become disengaged from the utopic idea of a counter culture which was nonconsumer and anti-materialistic, to focus now on the commoditization and "folklorization" of nature and culture. Rural communities have become self-employed ventures that provide large hotel agencies with exotic excursions and function as outsourcing companies that work for travel agencies and transportation owners. By taking into account the ecotourism as practiced in Huatulco, it has ceased to be an alternative concept and has become a complementary activity to the tourism industry.

Keywords: Ecotourism and neoliberalism. Exotism and touristic industries. Rural communities and folklorization. Huatulco Bays-Mexico.

\section{Introducción}

El ecoturismo ha sido, en un primer momento, sólo una preocupación de los activistas ecologistas ante los destrozos del turismo de masas, pero rápidamente la industria del turismo se dio cuenta del valor comercial o mercantil de la ecología, lo que la llevó a dar un giro de $180^{\circ}$ con respecto a sus posiciones anteriores. En efecto, en sus inicios había considerado a la ecología como un obstáculo, unas "piedritas en el camino" que retrasaban el desarrollo turístico, pero actualmente se ha convertido en un "plus" turístico que vuelve más atractivo cualquier lugar y, por ello, las agencias turísticas "organizan" a los pueblos cercanos a las playas como lugares de ecoturismo.

Analizando el caso de las Bahías de Huatulco, Oaxaca, se puede detectar cuatro tipo de actores interesados en promover políticas de turismo ecológico: 1) las comunidades campesinas que no alcanzan vivir de su trabajo agrícola y requieren otras formas de ingresos; 2) las agencias de turismo que comercializan las excursiones ecoturísticas, en este caso un paseo por las "cascadas mágicas" o bien un tour por "pueblos y tradiciones" o unos "paseos en cuatrimotos por la jungla"; 3) la Secretaría de turismo estatal que apuesta a la diversificación de ofertas turísticas para "ayudar" y arraigar a las comunidades en dificultades económicas y evitar una migración mayor; 4) y, a nivel nacional, el Gobierno Federal, cuyas políticas agrarias plantean y ofrecen el ecoturismo como "la" solución para remediar todos los males provocados por las 


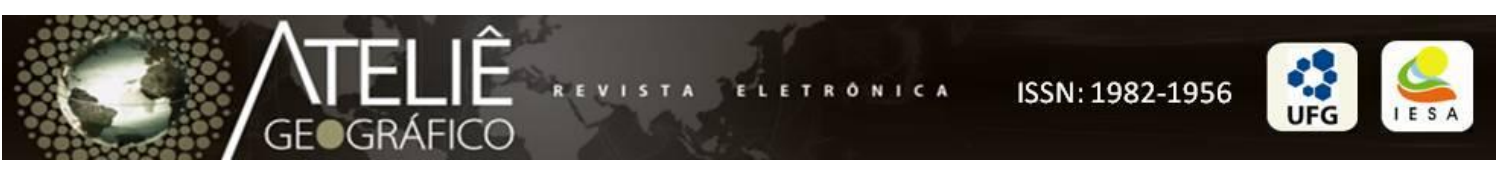

políticas de abandono del campo ejidal o comunitario, es decir, una barita mágica o una "aspirina para el campo", ya que no afronta las causas y sólo atenúa los efectos nocivos de las políticas económicas recientes. Por ello el gobierno ha integrado a la ecología en sus planes de desarrollo turístico.

\section{Turismo y Ecología en Huatulco}

Las Bahías de Huatulco fueron expropiadas (21,000 has) en 1984 como megaproyecto turístico. Al no tener el éxito previsto, Bahías tuvo que re-orientar sus targets. Al inicio se planeó como un resort muy exclusivo para turismo de altos recursos que emplearía mucha mano de obra en el sector servicios. Pero la exclusividad de un desarrollo de super lujo (5 estrellas con golf) muy alejado de todo centro urbano, que no puede ofrecer diversiones de vida nocturna a la juventud; para el cual, además, se privilegia el acceso por vía aérea en detrimento de un buen acceso carretero, fueron algunas de las razones que hicieron que Huatulco "no despegara" y que los hoteles de 5 estrellas (Sheraton, Camino Real, Maeva, ClubMed) fueran vendidos para volverse VTP (Viaje todo pagado).

Pero abrir Huatulco al turismo de masas no fue suficiente, y actualmente se multiplica el turismo de cruceros y se intenta promocionar el ecoturismo presentando a Huatulco como un paraíso. Este desarrollo planeado para ocupar nueve bahías quedó truncado, reduciéndose a sólo dos de ellas: Santa Cruz y Tangolunda. En las demás bahías sólo se otorgaron concesiones para la construcción de palapas a los habitantes originarios del municipio de Santa María. Por eso, cuando se vencieron los plazos de la expropiación y para evitar su reversión, - que obligaría a devolver las bahías no ocupadas a la comunidad de Santa María -, se creó un Parque Ecológico Nacional. Es el primer uso político que se dio a la ecología cuando sirvió para "taparle el ojo al macho" y no devolver las tierras comunales a sus legítimos poseedores. El segundo uso político es el actual ecoturismo.

En la urbanización de Bahías de Huatulco, Fonatur (Fondo Nacional para el Turismo) - la institución que la planeó y ejecutó - respetó los reglamentos internacionales en materia de ecología: prohibición de descarga de desagüe en las bahías y plantas de tratamiento de aguas negras, construcción de un pueblo de apoyo para 


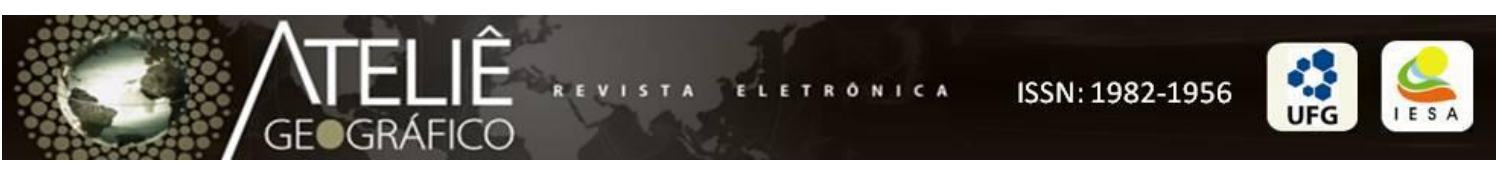

alojar a los trabajadores (llamado Crucecita y ubicado de espalda al mar, atrás de un cerro) y conducción de todas las instalaciones eléctricas por vía subterránea. En sus inicios, Bahías no dañó mucho al mar, hasta la fatídica construcción del muelle de Santa Cruz que destruyó muchos corales y, actualmente, los pocos corales sobrevivientes (en la playa de La Entrega) se ahogan bajo las arenas que remueven las hélices de los supercruceros (con capacidad para 3,500 personas) cuando atracan en el muelle o zarpan hacia alta mar.

En la urbanización local de Crucecita, se concedieron predios a los huatulqueños "expropiados", que de ese modo realizaron su reconversión de agricultores y pescadores a proveedores de servicios locales: taxis, lanchas, cuartos de hospedaje locales, tienditas misceláneas, fondas y cantinas. Se formó así una mini-clase media local. Actualmente los expropiados originarios organizados en cooperativas (de taxis, lanchas, palapas y, últimamente, gasolinerías) "rentan" sus concesiones y viven de sus rentas. Al mismo tiempo se mudaron a Santa Cruz los primeros inversionistas (de Puebla, Guerrero, Oaxaca y D.F.), cuyos negocios derivaban del negocio hotelero: constructoras, gerentes de bancos, concesionarios de casas de materiales y cervezas, hoteleros para turismo nacional fuera de las playas, agentes de turismo, restauranteros, comerciantes (dueños de tiendas de autoservicios, de farmacias y de negocios de bicicletas, etc.), agentes de bienes raíces, y así sucesivamente, que conformaron rápidamente una élite local que administra económica y políticamente el conjunto turístico de mano con Fonatur. Para todos los habitantes de Crucecita queda claro que Bahías sirve también en parte para el lavado de dinero, pero es un tema que mejor no se toca.

A partir de la expropiación del litoral, los habitantes del municipio de Santa María Huatulco quedaron divididos en cuatro grupos: 1) los expropiados (a su vez, subdivididos en tres comunidades: Santa Cruz, El Arenal y Coyula); 2) los huatulqueños habitantes de las comunidades serranas (agricultores y cafetaleros); 3) los habitantes de la cabecera municipal, así como las autoridades locales, que viven de la ubre de Fonatur; 4) los hijos de los expropiados que reclaman algunos beneficios del turismo, ya que se vieron privados de sus herencias. En esta partida de billar a cuatro bandas, ¿dónde quedó la bolita de la ecología?

\section{Una Ecología Mediterránea}


La zona hotelera se construyó siguiendo los parámetros urbanísticos internacionales. La "ecología" consistió en ocultar todo: cables de luz, tuberías de agua y de desagüe. Era también un requisito estético. El "paisaje" urbano se inventó trayendo pasto, cocoteros, buganvilias, laureles y cactus que fungieron como símbolos de sol, playa y exotismo. Del hábitat ecológico original sólo quedaron algunas rocas en los camellones y unas que otras iguanas, como mudos testigos de tiempos pasados. El paisaje turístico se volvió mediterráneo, lo que implica la obligación para los particulares de incluir en la arquitectura de sus casas unas bóvedas o cúpulas de estilo árabe. ¡La Meca en Huatulco en medio de jardines y campo de golf de verdes pastos, muy british!

De este modo, Bahías de Huatulco expresa toda la fantasía californiana y europea de un imaginario exótico y gozoso ligado al sueño de un eterno verano vacacional donde las Huris del paraíso coránico y las Evas cristianas hubiesen recreado su "paraíso en el Pacífico", libre de todo pecado y lejos de todo trabajo, con la sola obligación de disfrutar del día, - "carpe diem”, como diría el filósofo griego. Una ecología paradisiática pero muy alejada de la cruda realidad.

\section{La Práctica Ecologista}

Desde antes que naciera la pulcra Huatulco, una "buena onda" ecologista recorría la costa sur de Oaxaca, con Zipolite como epígono. Zipolite, una playa de difícil acceso, sirvió de refugio hippy con sus drogas como cantos de sirena y el beso mortal de sus olas, pero significó, ante todo, una gran rebeldía anti-consumista, un retorno a la naturaleza auténtica y sin maquillaje, una vuelta al hábitat natural. Después del 68, la utopía de poder educar a los hijos en armonía con la naturaleza, al margen de todo consumismo, llevó a algunas parejas a instalarse en un pueblito de pescadores cercano que, después de vivir de la matanza de tortugas hasta arriesgar su desaparición, se había convertido en su protector. Es así como el deseo de escapar de la cultura urbana tan materialista, llevó a redimir tanto al turista avecindado en Mazunte, como a sus habitantes convertidos de depredadores en salvadores de la fauna marina, mediante la construcción de un criadero/museo de la tortuga y una fábrica de productos cosméticos 
naturales. Fue la primera experiencia de "rentabilización" de la ecología. La ecología dejó de ser una mera reglamentación urbanística o una rebeldía anticonsumista para volverse fuente de empleo. Se inicia así la ecología como negocio bajo la forma comunitaria de cooperativismo.

Actualmente, Mazunte es un agregado de negocios particulares de renta de cabañas y ofertas de mala comida donde la ecología sirve de excusa y de máscara para el descuido. Se acabó el proyecto inicial de una arquitectura alternativa y natural: ahora los comuneros se han vuelto "hoteleros" y se esfuerzan por rentabilizar sus cabañas, muchas de ellas construidas de tabicón y techos de cartón. La ecología se volvió un lujo que las poblaciones locales y el turismo estacionario no pueden sufragar.

Por otra parte, el semi-sagrado camino a Punta Cometa - lugar de "adoración" del sol - se convirtió en un sacrílego desarrollo playero con sus casas de concreto. Sin embargo, quedó un núcleo de heroicos soñadores que viven alrededor del camino que lleva a Playa Mermejita y ofrecen su asesoría para servicios de masaje, venta de energía solar y/o de comida vegetariana. En torno a Mermejita se vende el sueño de Robinson Crusoe. Ahí también la ecología es negocio: la realidad se ha comido a la utopía, pero al menos no dañan a la naturaleza, más bien la protegen.

Es decir que al iniciar el siglo XXI, el concepto de ecología se ha desvinculado por completo de toda idea utópica de contra-cultura anti consumista y anti materialista. La ecología se limita ahora a controlar los daños de la urbanización. Bajo la rúbrica de "negocio", - "hay que vivir de algo" -, se juntaron turismo y ecología: los antiguos mochileros son ahora los promotores del turismo ecológico.

\section{¿Un Negocio Nuevo o un Nuevo Negocio? Ecoturismo en torno a Huatulco}

Como se mencionó al inicio, el proyecto turístico Bahías de Huatulco dividió a la comunidad de Santa María. Por un lado Fonatur, en su intento por diversificar la oferta turística y volverla más atractiva, ha fomentado el espejismo del ecoturismo entre las comunidades no expropiadas de la Sierra Sur. Por otro, las comunidades del litoral que fueron expropiadas en 1984 y que no han dejado de luchar para revertir el decreto expropiatorio, como las de los Bajos del Arenal y de Coyula, quieren asumir por sí mismas su propio proyecto de desarrollo turístico bajo forma de ecoturismo. Ahora 
bien, ¿quién va a financiar tales proyectos ecoturísticos? Las comunidades serranas que no fueron expropiadas pueden conseguir ayudas gubernamentales, ya que están en posesión de sus tierras, mientras que las comunidades de la costa despojadas por Fonatur no son personas morales ni sujetos de crédito. Todas sus tierras pertenecen a Fonatur y con ello perdieron todo derecho a cualquier ayuda económica que no sea canalizada mediante Fonatur. Pero solicitar la intermediación de Fonatur equivaldría a reconocer de facto la legalidad de la expropiación. Impensable. Por ahora no pueden concertar ningún préstamo ni ayuda, porque no tienen el respaldo de la tenencia de la tierra. Sus caminos de terracería tampoco son asfaltados por la Federación, ya que dependen de Fonatur, y sus habitantes rechazan la urbanización ofrecida por Fonatur porque sería reconocer de facto los derechos de Fonatur sobre sus comunidades. Por eso ellos dan mantenimiento a sus caminos gracias al tequio.

Por el contrario, las comunidades serranas que no fueron expropiadas obtuvieron dinero de la CDI (Comisión para el Desarrollo Indígena) en tiempos de Xóchitl Gálvez, es decir, entre el 2000 y el 2006, todo ello mediante la asesoría y la gestión del principal operador turístico de los cruceros que atracan en Huatulco dos veces a la semana. Se organizaron en una Asociación Civil llamada "Pueblos y Tradiciones de México", que funciona como una Cooperativa de producción agrícola y artesanal. Es un negocio redondo para el tour-operator (agencia de excursiones), que ha logrado que la CDI financie unas palapas turísticas con baños al amparo de los proyectos comunitarios y que los habitantes enseñen y expliquen a los "cruceristas" sus actividades productivas. La agencia sólo paga a los cooperativistas 10 pesos por turista, mientras que ella cobra en dólares la excursión sin ningún costo de inversión, ya que los gastos fueron cubiertos por la CDI.

Más retirados de la costa, los pueblos serranos del municipio de San Miguel del Puerto, dueños de unas cascadas, lograron también financiamiento de la CDI para la construcción de cabañas y de un mariposario, así como para la compra de bicicletas y la creación de viveros de plantas medicinales, todo esto con el propósito de reconvertirse al ecoturismo para atraer igualmente el turismo de Huatulco. Llama la atención que estas comunidades agrícolas serranas hayan entrado también en la esfera de atracción de Bahías: ¿realmente no hay salida para los campesinos fuera de Fonatur y las agencias que giran en su entorno como buitres (dispuestos a tragarse tanto al turista como al 
comunero)? Esto es lo que hace creer el gobierno porque le resulta muy conveniente que los comuneros dejen de desmontar el monte, ya que la calidad del café de los ranchos cafetaleros vecinos -denominado café de altura- depende de la humedad del suelo, y el desmonte perjudica el nivel de humedad de los terrenos circunvecinos. Entre maíz y café, se optó por proteger a este último, y la pantalla del ecoturismo permite conservar la vegetación serrana. La clientela de las cascadas "mágicas" no se recluta entre los cruceristas por falta de tiempo, - ya que éstas están ubicadas a hora y media de Bahías -, sino entre los turistas invernales (en su mayoría canadienses) que gozan de largas estadías en los hoteles o el turismo mexicano que llega en coche (muchos vienen de Salina Cruz). En el municipio de San Miguel del Puerto no sólo están las cascadas, sino también la ruta del café que comparte con Pluma Hidalgo (municipio de San Pedro Pochutla). Otro lugar añejo de turismo en lo alto de la montaña (muy vinculado a Zipolite), en la ruta que lleva de Pochutla a Oaxaca, es San José del Pacífico, gran lugar de "adoración" de los hongos alucinógenos.

En el litoral, los que eran pescadores se dedican ahora a organizar "tours" por el litoral; los cafetaleros trabajan de jardineros o veladores y los campesinos intentan seguir cultivando maíz, pero sus hijos prefieren emigrar.

\section{Migración y Ecoturismo: ¿Alternativas para Seguir Siendo Campesinos?}

El comité de Bienes Comunales de Santa María Huatulco por el período 20092011 está compuesto por representantes de varias comunidades del municipio (el Presidente es de los Bajos del Arenal, el Secretario de los Bajos de Coyula, el tesorero de San Francisco Limoncito), todos preocupados por desarrollar el ecoturismo como "otra manera" de aprovechar la tierra, de seguir siendo campesinos, particularmente para las comunidades serranas - tradicionalmente maiceras - que no fueron expropiadas, por lo cual no gozaron de ninguna concesión en Bahías. Intentan también convencer a sus vecinos para abandonar la forma de cultivar con base en el sistema de "roza y quema", ya que deforestan los cerros, pero ante la falta de apoyos gubernamentales para compra de fertilizantes - y los bajos salarios (150 pesos/día, que son 70 dólares semanales, mientras que del "otro lado" juntan 300 dólares semanales), se mantiene la práctica de "roza y quema". 


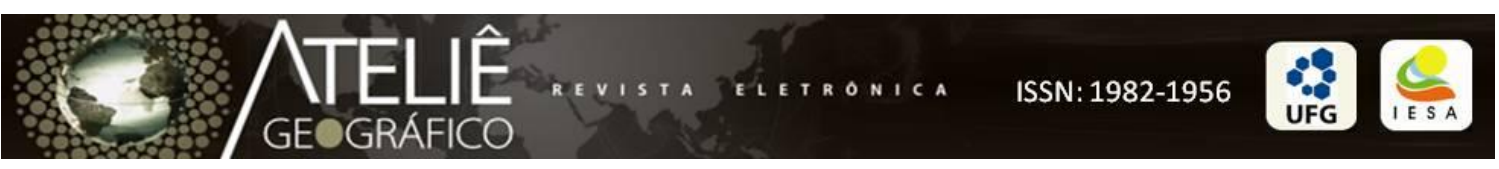

Para las comunidades serranas, el ecoturismo es la mejor vía para quedarse en sus tierras; sin embargo la migración temporal a los Estados Unidos es el único recurso para financiarse. De hecho, casi todos migran entre los 18 y 20 años, pero sólo $25 \%$ se quedan del otro lado, mientras que el $75 \%$ regresan, según explica Isaac Santana Aragón, de Limoncito. Los migrantes envían las remesas para construir su casa, para agrandar la parcela o simplemente para que su familia viva al día. Pero los mayores ya se cansaron de ese ir y venir (migran por temporadas de 3 o 4 años varias veces en sus vidas), ya no quieren "cruzar el desierto" y esperan que el ecoturismo - mediante la resurrección de antiguos oficios y sembradíos exóticos - pueda asegurarles un ingreso semi-fijo que complete lo que ganan con sus cultivos. Por ello crearon su cooperativa "Pueblos y Tradiciones de México" y la CDI dio a la asociación 1,500,000 pesos (información de Isaac Santana Aragón) para construir las palapas y los baños que se ven al frente de sus casas. La cooperativa Pueblos y Tradiciones organizó un jardín botánico en Piedra de Moro (pero en 2010 se salió), un plantío de nopales en Pueblo Viejo, y en San Francisco Limoncito una huerta de plátanos y otra de cítricos. Además fomentó artesanías de "palma mixteca" (que traen de Miahuatlán), así como la fabricación de adobe. Últimamente planea un recorrido en carreta de bueyes.

En los cerros que dominan Pueblo Viejo y San Francisco, llamados Cerros de Piedras Negras, dicen que hay "grandes piedras donde ahí vivía gente antes" y que "el 24 de diciembre se oyen voces". En los Cerros hay iguanas y venados, por lo cual la Cooperativa quisiera construir cabañas para promover paseos (en cuatrimotos...) por los cerros, pero se requiere financiamiento. La CDI ya lo otorgó. Ahora sólo resta tocar la puerta del futuro gobierno estatal electo el 4 de Julio del 2010 (la Coalición de Gabino Cué), que entrará en funciones al comienzo del 2011.

\section{¿A Quién Beneficia el Ecoturismo?}

¿Por qué esta fascinación por el ecoturismo? Porque conviene a las agencias de turismo, que son los intermediarios que se quedan con las ganancias ("el que parte, reparte y se queda con la mejor parte"); porque conviene a los hoteleros en la medida en que el turista se hospeda por más tiempo cuando tiene actividades alternativas al sol y playa, y porque, principalmente, conviene al Estado que de este modo se desentiende de todos sus compromisos de política agraria, ya que las comunidades salen de su esfera de 
responsabilidad económica (Procampo), quedando vinculadas a la CDI y a la Secretaría de Turismo.

Las comunidades funcionan entonces como empresas de outsourcing (subcontratación) que proporcionan distracción a los turistas, pero sin que los negocios hoteleros y de agencias de viaje tengan que invertir un peso: no corren ningún riesgo. Toda la inversión y el trabajo corren a cargo de las comunidades que, finalmente, sólo reciben migajas que deben aceptar por falta de otras alternativas (como tener su propio transporte y agencia). En consecuencia, el Estado ha dejado de apoyar al pequeño campesino y se limita a conminar a las comunidades a que desarrollen su "ecoturismo" como panacea a todos sus males. La CDI facilita fondos para resucitar oficios tradicionales, de tal manera que los turistas extranjeros puedan acudir en una excursión de tres horas a visitar "Pueblos y Tradiciones" cuando su crucero ancla en el muelle de Santa Cruz.

De este modo se ha montado todo un escenario folklórico donde algunas familias enseñan pequeños plantíos de nopales y unas matas de plátano en sus miniparcelas, porque el turista sólo conoce los cítricos y la "banana" sin distingo de especies, y se maravillan cuando descubren que hay una gran variedad de especies. Además, para ellos es toda una novedad conocer lo que es una carreta de bueyes, más algunas hortalizas y plantas medicinales. Así, una familia se dedica a tejer la "palma mazateca", es decir, canastitas, abanicos para el fogón y petates, mientras que otra se dedica a fabricar adobes (cuando en realidad su casa está construida de tabicones recubiertos con una mezcla de barro con silicón). Es así como se llega a montar un escenario que recurre a la cultura y la ecología para vender un "taco de ojo" o perlitas de vidrio al gringo. Pero ¿qué es lo que queda en México, ya que el turista ni consume los alimentos locales ni bebe las aguas de frutas? Nada... sólo unos campesinos ilusionados con la perspectiva de poder vivir algún día del ecoturismo y del turista, cuando realmente los esfuerzos y el trabajo están del lado de los comuneros, mientras que los beneficios se quedan para el intermediario, dueño de la agencia y de los vehículos. Al parecer, en toda la región serrana se considera que fuera del turismo no hay salvación. Pero, ¿acaso hay salvación con el turismo?

\section{Conclusiones}


Los pueblos serranos del municipio de Santa María Huatulco y los aledaños (San Miguel del Puerto, San Pedro Pochutla y Santa María Tonameca) sirven de respaldo en áreas de servicios a Bahías. Primero fueron integrados al desarrollo turístico como reserva de mano de obra $\mathrm{y}$, últimamente, sus paisajes agrestes sirven de atractivo turístico para diversificar la oferta turística huatulqueña y entretener "ecológicamente" a los paseantes. En efecto, para retener unos días más al turismo, se necesita diversificar la oferta, y la ecología es un tópico de moda que se vende bien. Como los cruceros requieren de excursiones cercanas, una agencia de excursiones ha construido un verdadero enclave de ecoturismo folklórico organizando las actividades susceptibles de gustarle al turista. Este promotor turístico ayudó a las comunidades a redactar sus proyectos de financiamiento, lo que lo posicionó ante ellas como su protector y benefactor. De esta manera se ha podido vincular directamente el ecoturismo con una estancia corta en Bahías.

Sin embargo, los pueblos quisieran arraigar por sí mismos a los turistas amantes de la naturaleza y romper el monopolio ejercido por Bahías. Para lograrlo están construyendo cabañas, ya que el ecoturismo proporciona al campesino otro medio u otra manera de vivir de su tierra, y para el turista evoca un retorno a la naturaleza y a formas sencillas de vida. Actualmente el ecoturismo huatulqueño se limita a los excursionistas de los hoteles o cruceros que buscan exotismo y folklore (nostalgia por las tradiciones), pero debería vincularse también con el turismo mochilero, alejado de Fonatur, que no viene a curiosear sino a compartir, tal como ocurre en los Ocho Pueblos Mancomunados de Oaxaca o la red de Turismo Indígena. El turismo mochilero busca integrarse a la cotidianeidad de la comunidad y establecer vínculos de igualdad; este turismo debe surgir como iniciativa de las comunidades mismas, es decir desde abajo y no como proyecto de Fonatur obnubilado con complacer al turista norteamericano. Las comunidades deben vivir para sí mismas, no para el turista.

Finalmente, cuando las decisiones sobre ecoturismo se toman entre dueños de agencias dejando una mínima parte a las decisiones locales, se está creando de hecho un caciquismo rural de nuevo cuño, en virtud del cual las comunidades están al servicio de la comercialización de una "tradición inventada". El cacique de nuevo cuño no requiere 
controlar las tierras o el acceso a ellas, sino solamente monopolizar el flujo de turistas que trae y lleva, comprando por diez pesos (en 2010) las sonrisas de los habitantes.

\section{Bibliografia}

ALBA RICO, Santiago. "Turismo: la mirada caníbal", Revista Archipiélago, n.68, España, 2005.

BOLTANSKI, L. y CHIAPELLO, E. El nuevo espíritu del capitalismo, España: Akal, 2002.

BOURDIEU, P. La distinción, Madrid: Taurus, 1991.

CASTELlANOS GUERRERO, A., MACHUCA, R. (comps). Turismo, identidades y exclusión, México: UAM, 2008.

DA JANDRA, L.Entrecruzamientos, Oaxaca: Almadía, [1985] 2005.

Huatulqueños, Oaxaca: Almadía, [1991] 2005

. Samahua, Oaxaca: Almadía, .[1997] 2005.

DESFORGES, L. Tourism: A Cultural Economy, London: Sage Publications, 2010.

DOMINGUEZ-LICONA, J.M. Cacaluta, Oaxaca: Universidad del Mar, 2008.

GASCÓN, J. El turismo en la cooperación internacional, Barcelona: Icaria, 2009.

GLADSTONE, D. From Pilgrimage to Package Tour, New York: Routledge, 2005.

GOTTDIENER, M. New forms of Consumption, Maryland: Rowman and Littlefield, 2000.

GREENWOOD, D. "Culture by the pound: an anthropological perspective on tourism as cultural commodization" en Hosts and Guests, Philadelphia: University Press, 1989.

HALL, M. Ecotourism or Ecological Imperialism, Revista Geographical, 67 (2), 1995.

LASH, S. y URRY, J. Economias de Signos y Espacio, Buenos Aires: Amorrortu, 1998.

LIPOTVETSKY, G. La felicidad paradójica, Barcelona: Anagrama, 2007.

MAcCANNELL, D. El turista. Una nueva teoría de la clase ociosa, Barcelona: Melusina, 2000.

MALDONADO, Carlos. Turismo comunitario, Doc.73, OIT, 2005. 
MONTERROSO SALVATIERRA, N. e ZIZUMBO VILLAREAL, L. Contra la domesticación del turismo, México: Miguel Angel Porrúa, 2010.

MOWFORTH, M. y MUNT, I. Tourism and sustainability: New Tourism in the Third World, London: Rouledge, [1998] 2009.

PEDREÑO, A. y CASTELLANOS, M.L. Los nuevos braceros del ocio, Madrid: Miño y Dávila, 2006.

\section{Agradecimientos}

Agradezco el apoyo del proyecto CONACYT, número 83606, "Cultura, identidades y relaciones interétnicas en ciudades turísticas internacionales en México" y al programa PAPITT (proyecto IN304609), "Procesos transnacionales: migración, turismo y relaciones interétnicas" para la elaboración de este resultado de investigación.Apresentado en el III Coloquio Internacional Ciudades del Turismo: lugares, prácticas y representaciones.Hermosillo, Mexico, 2010.

Recebido para publicação em outubro de 2010 Aprovado para publicação em julho de 2011 